\title{
Coupled Fluid Flow and Geomechanics in Fractured Reservoir Study
}

\author{
Chen, Her-Yuan; Harstad, Hugo; and Teufel, Lawrence W. \\ New Mexico Institute of Mining and Technology, Socorro, New Mexico, USA
}

Paper presented at the 5th European Conference on the Mathematics of Oil Recovery, Leoben, Austria, 3-6 Sept. 1996

\begin{abstract}
The purpose of this study is to formulate a coupled fluid-flow and geomechanics model of a naturally fractured reservoir. Fluid flow is modeled within the context of dualporosity (more generally, overlapping-continuum) concept while geomechanics is modeled following Biot's isothermal, linear poroelastic theory. The development follows along the line of the conventional and existing porous fluid-flow modeling. The commonly used systematic fluid-flow modeling is therefore preserved. We show how the conventional fluid-flow dual-porosity formulations are extended to a coupled fluid-flow-geomechanics model. Interpretation of pore volume compressibilities (fractures and matrix blocks) and the associated effective stress laws are identified to be the most critical coupling considerations. Several limiting cases including singleporosity, single-permeability/dual-porosity, and incompressible solid are analyzed.
\end{abstract}

\section{INTRODUCTION}

Geomechanics is particularly important in petroleum reservoir management of naturally fractured reservoirs [Teufel et al., 1993]. Economical petroleum production from most naturally fractured reservoirs relies on the fracture permeability (including magnitude and orientation of anisotropy). Natural fractures basically are the product of evolving rock stress state. Therefore any disturbance of the stress field, such as due to fluid production/injection, can affect the existing fractures (e.g., opening, closure, reorientation) and the associated reservoir performance. A coupled fluid-flow and geomechanics model thus provides a rational tool for a better understanding and management of a naturally fractured reservoir.

The theory describing fluid-solid coupling was first presented by Biot $[1941,1955,1956]$ in which mechanical issues were emphasized over the fluid flow issues. Because of this, Biot's theory is less compatible with the conventional fluid-flow models (without geomechanics considerations) in terms of concept understanding, physical interpretation of geomechanical parameters involved (e.g., rock compressibilities), and computer code upgrading. These issues, however, can be resolved if Biot's theory is reinterpreted and reformulated along the line of conventional fluid-flow modeling as done by Geertsma [1957] and Verruijt [1969], and recently by Chen et al. [1995]. In essence, these reformulations provide better "compatibility" and "expandability" to the existing fluidflow knowledge and models.

The original Biot's theory is a single-fluid/single-solid model, i.e., a single-porosity type of model from a fluidflow point of view. Naturally fractured reservoirs are often modeled by the dual-porosity (overlapping continuum) type of concept developed by Barenblatt et al. [1960]. Models incorporating both Biot's poroelastic theory and Barenblatt et al.'s dual-porosity concept have been presented by several authors. These models can be classified into two types depending on the approach taken. The first approach is based on mixture theory and was adopted by Wilson and Aifantis [1982], Beskos and Aifantis [1986], and Bai et al.'s [1993]. Two related features of the resulting formulations are: (i) all the fluid-flow equations in a "mixture" have the same functional form as that of a single-porosity if the fluid exchange term is dropped, (ii) phenomenological coefficients are proposed firstly and their 
physical interpretations are deduced, if necessary, after the completion of the formulation.

Item (i) implies that the changes of stress-dependent rock properties in a continuum are independent of other continua to be mixed. This in turn may cause difficulty for the later physical interpretation (see item (ii)) and even inconsistency with the geomechanical equations adopted.

The second approach follows the route of conventional fluid-flow modeling. Coupling of geomechanics is identified through stress-dependent rock properties and during the development process. Interpretations of the stress-dependent properties is therefore critical to achieving a proper coupling. This approach was adopted by Duguid and Lee [1977], Valliappan and Khalili-Naghadeh [1990], and Khalili-Naghadeh and Valliappan [1991].

Duguid and Lee [1977] emphasized the fluid-flow aspects while neglecting most of the issues of geomechanical coupling. They assumed an incompressible solid and the pore volume compressibilities (fracture and matrix blocks) were represented by the fluid compressibility. Thus, no explicit rock compressibilities (solid, pore, or bulk) appear in their fluid-flow equations. The only coupling term evolved (the divergence of solid velocity) was dropped eventually in their numerical implementation.

Explicit rock compressibilities were considered by Valliappan and Khalili-Naghadeh [1990] and KhaliliNaghadeh and Valliappan [1991]. Certain rock compressibilities were involved but are not clearly defined and lack justifications.

In view of the above discussions, uncertainties still exist regarding the modeling of the coupled fluid-flow and geomechanics processes in a naturally fractured reservoir, as well. as in the simplest fluid-rock setting (singlefluid/elastic-rock). The purpose of this paper is to analyze and formulate the coupled fluid-flow and geomechanical modeling within the framework of the dual-porosity concept. Identification of the linkages and consistent interpretations between the flow and deformation fields are emphasized. Such an emphasis is necessary to maintain a consistent design and usage of laboratory data for the simulation study of field problems.

\section{BASIC PRINCIPLES}

Basic principles governing isothermal, single-phase fluid flow in a deformable fractured medium are described in this section. Biot's [1941, 1955, 1956] poroelastic theory describing fluid-solid coupling of a single-porosity system will be extended to a dual-porosity system. The derivations to be presented, as pointed out before, are intended to follow along the line of existing fluid-flow modeling.
Dual-Porosity. The fractured medium is modeled in the context of dual-porosity (overlapping-continuum) concept [Barenblatt et al., 1960]. Specifically, two overlapping continua are considered: matrix-blocks (primary pores) and fractures (secondary pores). Each continuum possesses its own fluid-pressure field. To consider rock deformation, the rock stress field is further superimposed on the two fluidpressure fields.

We will use subscript $n=1$ to denote the matrix-block (primary pores) while $n=2$ for the fractures (secondary pores), and the subscript $s$ for the solid phase. The bulk fractured medium, $V_{b}$, thus is viewed to comprise three components:

$$
V_{b}=V_{p 1}+V_{p 2}+V_{s}
$$

where $V_{p 1}$ is the pore volume of matrix blocks, $V_{p 2}$ is the pore volume of fractures, and $V_{s}$ is the solid volume. In terms of porosity, Eq. 1 becomes

$$
1=\phi_{1}+\phi_{2}+\left(V_{s} / V_{b}\right)
$$

where $\phi_{1}=V_{p 1} / V_{b}$ and $\phi_{2}=V_{p 2} / V_{b}$. Distinction of two types of porosities is the first and the most basic concept in a dual-porosity (dual-continuum) description. Consideration of fluid-flow and rock-stress associated with these two porosities and the host rock frame are discussed next.

Fluid Flow. The three basic principles of fluid flow in porous media are: mass conservation, Darcy's law; and equation of state. Mathematically, these are:

Mass conservation:

Fluid: $\nabla \cdot\left(\rho \phi_{n} \mathbf{v}_{n}\right)+\frac{\partial\left(\phi_{n} \rho_{n}\right)}{\partial t}+(-1)^{n} \rho \Gamma=0$

Solid: $\nabla \cdot\left[\rho_{s}\left(1-\phi_{t}\right) \mathbf{v}_{s}\right]+\frac{\partial\left[\left(1-\phi_{t}\right) \rho_{s}\right]}{\partial t}=0$

Darcy's law:

$$
\phi_{n}\left(\mathbf{v}_{n}-\mathbf{v}_{\mathbf{s}}\right)=-\frac{k_{n}}{\mu} \nabla p_{n},
$$

Equation of state (isothermal fluid compressibility):

$$
c_{n}=\frac{1}{\rho} \frac{\partial \rho}{\partial p_{n}} .
$$


In Eqs. 3 through 6, $\rho$ is the fluid density, $\mathbf{v}_{n}(n=1,2)$ is the $n$-th fluid velocity vector, $\phi_{n}$ is the $n$-th effective porosity, $\phi_{t}$ is the total effective porosity (i.e., $\phi_{t}=\phi_{1}+\phi_{2}$ ). The solid counterparts for density and velocity are $\rho_{s}$ and $\mathbf{v}_{s}$, respectively. $p_{n}$ is the $n$-th fluid pressure, $k_{n}$ is the $n$-th permeability, $\mu$ is the fluid viscosity, $c_{n}$ is the $n$-th fluid compressibility, $\Gamma$ is the volumetric exchange rate (interporosity flow, transfer rate) between fractures and matrix blocks per unit bulk volume, and $t$ is time. Also, $\nabla$ and $\nabla$. denote gradient and divergence, respectively.

The effective porosity reflects interconnected and mobile fluid-filled pores. The term "solid phase" as defined in this study includes both real solid grains and nonconnected (isolated) pores.

The fluid density, $\rho$, represents the actual microscopic density, i.e., fluid mass per unit fluid volume. $\rho \phi_{n}$ represents the apparent macroscopic fluid density, i.e., fluid mass per unit bulk volume. Similarly, $\rho_{s}$ and $\rho_{s}\left(1-\phi_{t}\right)$ are the actual microscopic solid density and apparent macroscopic density, respectively.

Both fluid velocity $\mathbf{v}_{n}$ and solid velocity $\mathbf{v}_{s}$ are local volume averaged values with respect to a stationary coordinate frame. $\phi_{n} \mathbf{v}_{n}$ represent the fluid bulk volumetric flux (fluid-flow rate per unit bulk area). Similarly, $\left(1-\phi_{t}\right) \mathbf{v}_{s}$ is the solid bulk volumetric flux. Note that for a deformable porous medium Darcy's law is expressed as fluid velocity relative to the moving solid as shown by Eq. 5 (due to Gersevanov in 1934 as cited by Biot [1955] and Verruijt [1969]). The condition for a nondeformable (stationary) medium is $\mathbf{v}_{s}=0$. Gravity effects are not considered.

Fluid density and viscosity are assumed to be a function of fluid pressure only, i.e., $\rho \equiv \rho(p)$ and $\mu \equiv \mu(p)$. For constant compressibility $c_{n}$, Eq. 6 can be integrated to give $\rho=\rho_{0} \exp \left[c_{n}\left(p_{n}-p_{0}\right)\right]$ where $\rho_{0}$ and $p_{0}$ are reference density and reference pressure, respectively. Rock properties are a function of rock stresses and fluid pressures. The rock compressibility will be introduced in the next section.

The interporosity-flow term, $\Gamma$ (see Eq. 3), is another unique aspect of the dual-porosity concept. This term, however, is also one of the major uncertainties in any dualporosity-based (also multi-porosity-based) model. The uncertainty is primarily due to the difficulty in describing the configuration, distribution, and connectivity of the fracture and matrix blocks. In the most general form, $\Gamma$ is a function of both fluid pressures in the matrix-blocks and fractures, i.e., $\Gamma \equiv \Gamma\left(p_{1}, p_{2}\right)$. Description of $\Gamma$ may range from a simple pressure-difference-controlled mechanism, i.e., $\Gamma \equiv \Gamma\left(p_{1}-p_{2}\right)$ [Barenblatt et al., 1960] to a more complicated history-dependent mechanism (see, e.g., Chen et al. [1990]). Because of the inherent uncertainty of $\Gamma$ and the emphasis of this study to incorporate the geomechanics into a dual-porosity concept, we will not specify the exact functional form of $\Gamma$ in this paper other than making the above general comments.

Geomechanics. Isothermal, perfect elastic medium (in the sense of linear, reversible, and non-retarded mechanical behavior) with small strains is considered. Stress and strain are positive in tension, whereas fluid pressure is positive for compression. The three basic principles of poroelastic theory are: stress equilibrium, strain-displacement, and strain-stress-pressure relations. They may be viewed as being parallel with the mass balance, Darcy's law, and equation-of-state of the fluid-flow modeling (see Eqs. 3 through 6). Mathematically, these are:

Stress equilibrium (quasi-static): (6 equations)

$$
\sum_{j=1}^{3} \frac{\partial \sigma_{i j}}{\partial x_{j}}=0 ; \quad \sigma_{i j}=\sigma_{j i},
$$

Strain-displacement relation : (6 equations)

$$
\varepsilon_{i j}=\frac{1}{2}\left(\frac{\partial u_{i}}{\partial x_{j}}+\frac{\partial u_{j}}{\partial x_{i}}\right),
$$

Strain-stress-pressure : (6 equations),

$$
\begin{aligned}
& \varepsilon_{i i}=\frac{1}{E}\left[\sigma_{i i}-v\left(\sigma_{j j}+\sigma_{k k}\right)\right]+\frac{\alpha_{1} p_{1}+\alpha_{2} p_{2}}{3 K_{b}}, \\
& \varepsilon_{i j}=\sigma_{i j} /(2 G), \quad(i \neq j) .
\end{aligned}
$$

In Eqs. 7 through 9, $\varepsilon_{i j}$ and $\sigma_{i j}$ are the components of bulk strain tensor and total stress tensor, respectively, $u_{i}$ is the component of solid displacement vector $\mathbf{u}\left(u_{x}, u_{y}, u_{z}\right), E$, $G(=E /[2(1+v)])$, and $v$ are Young's modulus, shear modulus, and Poisson's ratio for the solid skeleton under drained conditions, respectively, $K_{b}\left(=1 / c_{b}\right)$ is the drained jacketed bulk modulus of the fractured rock, and $\alpha_{n}$ is the $n$-th effective stress coefficient. More detailed definitions of $\alpha_{n}$ is discussed in Appendix A. Body forces and inertial effects are neglected in Eq. 7. Small strains are implied in Eq. 8.

Note that fluid pressure $p_{n}$ affects normal strains only and in the same manner due to the assumption of isotropy (see Eq. 9a). Shear strains are a function of shear stresses and are independent of fluid pressure (Eq. 9b). Eq. 9a, which includes the effects of two fluid-pressure fields, is a natural extension of the Biot's original single-pressure-field formulation. Basically, $\alpha_{n}$ determines the relative 
contribution of fluid-pressures on the elastic bulk behavior of a fractured medium.

It is more convenient to express stress in terms of strain because the total stress satisfies the equilibrium equation (Eq. 7). Solving Eq. 9 for stress gives

$$
\sigma_{i j}=2 G \varepsilon_{i j}+\lambda e \delta_{i j}-\left(\alpha_{1} p_{1}+\alpha_{2} p_{2}\right) \delta_{i j},
$$

where $\delta_{i j}$ is Kronecker's delta $\left(\delta_{i j}=1\right.$ for $i=j, \delta_{i j}=0$ for $i \neq j$,), and $\lambda$ is the Lame's constant (see Nomenclature). Adding the three equations given by Eq. $9 \mathrm{a}$ or 10 results in

$$
e=\frac{\sigma_{m}+\alpha_{1} p_{1}+\alpha_{2} p_{2}}{\lambda+(2 / 3) G}=\frac{\sigma_{m}+\alpha_{1} p_{1}+\alpha_{2} p_{2}}{\bar{K}_{b}}
$$

where $e\left(=\varepsilon_{x x}+\varepsilon_{y y}+\varepsilon_{z z}\right)$ is the dilatation or volume strain of the solid skeleton and $\sigma_{m}\left[=\left(\sigma_{x x}+\sigma_{y y}+\sigma_{z z}\right) / 3\right]$ is the mean normal total stress.

The solid displacement velocity $v_{s}$ and the volume strain $e$ are related to solid displacement vector $\mathbf{u}$ by

$$
\mathbf{v}_{s}=\partial \mathbf{u} / \partial t ; \quad e=\nabla \cdot \mathbf{u}
$$

The second expression, $e=\nabla \cdot \mathbf{u}$, may be established from Eq. 8. From Eqs. 4 and 12, it can be shown that the divergence of solid velocity $\nabla \cdot v_{s}$ is related to the volume strain $e$ by

$$
\nabla \cdot \mathbf{v}_{s}=\frac{\mathrm{d} e}{\mathrm{~d} t}=\frac{1}{V_{b}} \frac{\mathrm{d} V_{b}}{\mathrm{~d} t} ; \quad \mathrm{d} e=\frac{\mathrm{d} V_{b}}{V_{b}}
$$

It will be shown later that the first expression in Eq. 13 basically is a statement of mass conservation. The second expression provides a straightforward interpretation of the volume strain $e$.

\section{FIELD EOUATIONS}

In this section, coupled field equations are derived from the basic principles presented before. Terms which are critical in coupling are also identified.

Fluid Pressure Equation. Introducing Darcy's law (Eq. 5) into Eq. 3 gives

$$
\nabla \cdot\left(\rho \frac{k_{n}}{\mu} \nabla p_{n}\right)=\frac{\mathrm{d}\left(\phi_{n} \rho\right)}{\mathrm{d} t}+\phi_{n} \rho \nabla \cdot \mathbf{v}_{s}+(-1)^{n} \rho \Gamma,
$$

where $\mathrm{d}(\cdot) / \mathrm{d} t$ is the material derivative with respect to a moving solid defined as

$$
\frac{\mathrm{d}(\cdot)}{\mathrm{d} t}=\frac{\partial(\cdot)}{\partial t}+\mathrm{v}_{s} \cdot \nabla(\cdot)
$$

Eq. 15 links the material derivative, which is a Lagrangian concept, to a spatial or Eulerian description. Note that for nondeformable media the material derivative is equivalent to a partial derivative since $\mathbf{v}_{s}=0$.

Expanding the right-hand-side of Eq. 14 results in

$$
\begin{aligned}
\nabla \cdot\left(\rho \frac{k_{n}}{\mu} \nabla p_{n}\right) & =\phi_{n} \rho\left(\frac{1}{\rho} \frac{\mathrm{d} \rho}{\mathrm{d} t}+\frac{1}{\phi_{n}} \frac{\mathrm{d} \phi_{n}}{\mathrm{~d} t}+\nabla \cdot \mathbf{v}_{s}\right) \\
& +(-1)^{n} \rho \Gamma \ldots \ldots \ldots \ldots \ldots \ldots \ldots \ldots \ldots \ldots \ldots \ldots \ldots \ldots \ldots \ldots \ldots \ldots
\end{aligned}
$$

Expanding the first term of the solid mass balance equation, Eq. 4, and applying Eq. 15 gives

$$
\nabla \cdot \mathbf{v}_{s}=-\frac{1}{\left(1-\phi_{t}\right) \rho_{s}} \frac{\mathrm{d}\left[\left(1-\phi_{t}\right) \rho_{s}\right]}{. \mathrm{d} t}
$$

For a constant solid mass and noting that $\phi_{t}=V_{p t} / V_{b}$ and $V_{b}=V_{p t}+V_{s}$ where $V_{p t}, V_{s}$ and $V_{b}$ are the total pore (fractures and matrix blocks), solid, and bulk volumes, respectively, Eq. 17 is equivalent to

$$
\nabla \cdot \mathbf{v}_{s}=\frac{1}{V_{b}} \frac{\mathrm{d} V_{b}}{\mathrm{~d} t}
$$

Thus, the divergence of solid velocity simply reflects the rate of change of bulk volume. Eq. 18 combined with Eq. 12 leads to Eq. 13. From Eq. 18, $v_{s}=0$ (nondeformable) implies $\mathrm{d} V_{b}=0$.

Applying the relation $\mathrm{d} \phi_{n} / \phi_{n}=\mathrm{d} V_{p n} / V_{p n}-\mathrm{d} V_{b} / V_{b}$ and Eq. 18 to Eq. 16 results in

$$
\nabla \cdot\left(\rho \frac{k_{n}}{\mu} \nabla p_{n}\right)=\rho \phi_{n}\left(\frac{1}{\rho} \frac{\mathrm{d} \rho}{\mathrm{d} t}+\frac{1}{V_{p n}} \frac{\mathrm{d} V_{p n}}{\mathrm{~d} t}\right)+(-1)^{n} \rho \Gamma .
$$

Note that Eqs. 16 and 19 are identical to those derived for a single-porosity system [Chen et al., 1995] if the $\Gamma$ term is dropped. Also, these two equations with $\mathrm{d}(\cdot) / \mathrm{d} t$ replaced by $\partial(\cdot) / \partial t$ would be the same results obtained from Eqs. 3 through 5 with $v_{s}=0$ (nondeformable). The righthand-side of Eq. 19 basically represents the rate of change of fluid density and pore volume. The change of fluid density is related to the fluid compressibility $c_{n}$ by the following relations: 


$$
c_{n} \frac{\partial p_{n}}{\partial t}=\frac{1}{\rho} \frac{\partial \rho}{\partial t} ; \quad c_{n} \nabla p_{n}=\frac{1}{\rho} \nabla \rho ; \quad c_{n} \frac{\mathrm{d} p_{n}}{\mathrm{~d} t}=\frac{1}{\rho} \frac{\mathrm{d} \rho}{\mathrm{d} t} .
$$

Since the fluid density is a function of fluid pressure only, interpretation of the change of pore volume thus is crucial in coupling fluid-flow and geomechanics.

As discussed in Appendix $\mathrm{B}$, the change of pore volume, $\mathrm{d} V_{p n} / V_{p n}(n=1,2)$, are proposed to be

$$
\frac{\mathrm{d} V_{p 1}}{V_{p 1}}=\alpha_{1} \frac{c_{b}^{*}}{\phi_{1}}\left(\mathrm{~d} \sigma_{m}+\beta_{1} \mathrm{~d} p_{1}+\beta_{2} \mathrm{~d} p_{2}\right),
$$

and

$$
\frac{\mathrm{d} V_{p 2}}{V_{p 2}}=\left(1-\frac{c_{b}^{*}}{c_{b}}\right) \frac{c_{b}}{\phi_{2}}\left(\mathrm{~d} \sigma_{m}+\beta_{1} \mathrm{~d} p_{1}+\beta_{2} \mathrm{~d} p_{2}\right)
$$

Here, $\alpha_{1}$ is the effective stress coefficient associated with $p_{1}$ for the process of bulk volume change (see Eqs. A-7 and A8) while $\beta_{n}$ is the effective stress coefficients associated with $p_{n}$ for the process of pore volume change (see Eqs. B-8 and $B-10), c_{b} *$ is the bulk compressibility for rocks without fractures, $c_{b}$ is the bulk compressibility for fractured rocks. Eq. 21 maintains the functional form of a single-porosity system [Chen et al., 1995] and can be reduced to that of a single-porosity system by properly evaluating the parameters involved (see Eq. B-12).

Introducing Eqs. 20 and 21 into Eq. 19 results in

$$
\begin{aligned}
& \nabla \cdot\left(\frac{k_{1}}{\mu} \nabla p_{1}\right)=a_{11} \frac{\partial p_{1}}{\partial t}+a_{12} \frac{\partial p_{2}}{\partial t}+a_{13} \frac{\partial \sigma_{m}}{\partial t}-\Gamma \\
& \nabla \cdot\left(\frac{k_{2}}{\mu} \nabla p_{2}\right)=a_{21} \frac{\partial p_{1}}{\partial t}+a_{22} \frac{\partial p_{2}}{\partial t}+a_{23} \frac{\partial \sigma_{m}}{\partial t}+\Gamma
\end{aligned}
$$

where

$$
\begin{aligned}
& a_{11}=\phi_{1} c_{1}+\alpha_{1} \beta_{1} c_{b}^{*}, \\
& a_{12}=\alpha_{1} \beta_{2} c_{b}^{*}, \\
& a_{13}=\alpha_{1} c_{b}^{*}, \\
& a_{21}=\left(1-\frac{c_{b}^{*}}{c_{b}}\right) \beta_{1} c_{b},
\end{aligned}
$$

$$
a_{22}=\phi_{2} c_{2}+\left(1-\frac{c_{b}^{*}}{c_{b}}\right) \beta_{2} c_{b},
$$

and

$$
a_{23}=\left(1-\frac{c_{b}^{*}}{c_{b}}\right) c_{b}
$$

Two assumptions have been imposed in deriving Eq. 22 . First, the material derivatives can be approximated by the partial derivative, i.e., $\mathrm{d}(\cdot) / \mathrm{d} \cong \partial(\cdot) / \partial t$. This is equivalent to neglecting the dot product term in Eq. 15 by assuming $\nabla_{s} \cdot \nabla(\cdot)<<\partial(\cdot) / \partial t$. Second, the quadratic term $c_{n}(\nabla p)^{2}$ (which is also a dot product) may be neglected compared to the other terms.

Eliminating $\sigma_{m}$ between Eqs. 11 and 23 results in

$$
\begin{aligned}
& \nabla \cdot\left(\frac{k_{1}}{\mu} \nabla p_{1}\right)=b_{11} \frac{\partial p_{1}}{\partial t}+b_{12} \frac{\partial p_{2}}{\partial t}+b_{13} \frac{\partial e}{\partial t}-\Gamma, \\
& \nabla \cdot\left(\frac{k_{2}}{\mu} \nabla p_{2}\right)=b_{21} \frac{\partial p_{1}}{\partial t}+b_{22} \frac{\partial p_{2}}{\partial t}+b_{23} \frac{\partial e}{\partial t}+\Gamma,
\end{aligned}
$$

where

$$
\begin{aligned}
& b_{11}=\phi_{1} c_{1}+\alpha_{1}\left(\beta_{1}-\alpha_{1}\right) c_{b}^{*}, \\
& b_{12}=\alpha_{1}\left(\beta_{2}-\alpha_{2}\right) c_{b}^{*} \text {, } \\
& b_{13}=\alpha_{1} \frac{c_{b}^{*}}{c_{b}},
\end{aligned}
$$$$
b_{21}=\left(1-\frac{c_{b}^{*}}{c_{b}}\right)\left(\beta_{1}-\alpha_{1}\right) c_{b}
$$$$
b_{22}=\phi_{2} c_{2}+\left(1-\frac{c_{b}^{*}}{c_{b}}\right)\left(\beta_{2}-\alpha_{2}\right) c_{b}
$$

and

$$
b_{23}=1-\frac{c_{b}^{*}}{c_{b}} .
$$

Eq. 24 is the fluid-pressure equation with an explicit rock deformation effect described by the time rate change of volume strain. This deformation effect has to be resolved through the geomechanical consideration and is discussed next. 
Solid Displacement Equation. The total stresses given by Eq. 10 must satisfy the equilibrium relation specified by Eq. 7. Introducing Eq. 10 into Eq. 7 and applying straindisplacement relation (Eq. 8) give

$$
G \nabla^{2} u_{i}+(G+\lambda) \frac{\partial e}{\partial x_{i}}=\alpha_{1} \frac{\partial p_{1}}{\partial x_{i}}+\alpha_{2} \frac{\partial p_{2}}{\partial x_{i}},
$$

where constant values of $G, \lambda$, and $\alpha_{n}$ are assumed. These are three equations in $x, y$, and $z$. Note that $G+\lambda=G /(1-2 v)$. Eqs. 24 and 26 are five equations in five unknowns, $p_{1}, p_{2}$, $u_{x}, u_{y}$, and $u_{z}$. (Note that $e=\nabla \cdot \mathbf{u}$, see Eq. 12.)

By adding the three equations of Eq. 26, a compact representation is

$$
G \nabla^{2} \mathbf{u}+(G+\lambda) \nabla \nabla \cdot \mathbf{u}=\alpha_{1} \nabla p_{1}+\alpha_{2} \nabla p_{2} .
$$

Eqs. 24 and 27 forms a system with basic variables of scalar $p_{n}$ and vector $\mathbf{u}$. (Again note that $e=\nabla \cdot \mathbf{u}$.)

In summary, we have shown, starting from the basic principles, how the fluid-flow and geomechanics are coupled within the framework of dual-porosity concept. The coupled equations are presented by Eqs. 24 and 27 with variables $p_{n}$ and $\mathbf{u}$. This coupled system governs the time history of the fluid-pressure and rock deformation fields.

\section{DISCUSSIONS}

The presented derivations disclose that the changes of pore volumes (fracture and matrix blocks) are the most important considerations with respect to the mutual impacts of fluid-flow and geomechanics (see Eq. 19). This observation is also true for a single-porosity system [Chen et al. 1995] and is expected to be true for any overlappingcontinuum-based multi-porosity system. In fact it is sufficient to say that the differences between all the published models can be traced to the different interpretations of the pore volume changes.

Interpretation of pore volume changes, however, is difficult within the context of dual-porosity concept. In our approach, the functional form of the volume changes and the associated effective stress laws of a single-porosity system are extended heuristically to a dual-porosity system with the constraint that the dual-porosity system must be able to collapse to a single-porosity system. Thus, the issue (interpretation of pore volume changes) is transferred to the definitions of effective stress laws.

It is not difficult to image that attempting to define an exact and unique effective stress law is an extremely difficult task, given the inherently complex nature of a natural porous fluid-bearing rock (e.g., pore structure, rock constituents, anisotropy, nonhomogeneity, multiple fluid phases, etc.) and the manner in which real fractured rock masses are conceptualized in a dual-porosity concept. In this respect, we tried to identify the correspondence of effective stress coefficients, $\alpha_{n}$ (bulk volume) and $\beta_{n}$ (pore volumes), between ours and the models of Wilson and Aifantis [1982] and Bai et al. [1993] because of their different approaches. The results by comparing Wilson and Aifantis [1982] are: (i) $\alpha_{n}(n=1,2)$ are the same; and (ii) no correspondence can be established for $\beta_{n}(n=1,2)$, because of the different approaches and assumptions involved. We noted that Bai et al. [1993] assumed that the bulk compressibilities of fractured rock is the same as that of a rock without fractures. We believe this assumption is unrealistic and no further comparison is pursued.

Several limiting systems can be deduced from the previously derived results and are discussed next.

Single-Porosity System. The single-porosity system also implies a single set for the permeability field. The reduction process can be achieved by letting either the fracture volume or matrix pore volume shrink to zero, or by letting the two fluid-pressures equal.

If we let the fracture volume shrink to zero then $\phi_{2}=0$, $k_{2}=0$, and $c_{b}=c_{b}{ }^{*}$. It follows that $\alpha_{2}=\beta_{2}=0$ (see Eqs. A-9b and $\mathrm{B}-11 \mathrm{~b}$ ), $b_{12}=b_{21}=b_{22}=b_{23}=0, b_{13}=\alpha_{1}, b_{11}$ is still given by Eq. $25 a$, and $\Gamma=0$. Eqs. 24 and 26 are then reduce to

$$
\begin{aligned}
& \nabla \cdot\left(\frac{k_{1}}{\mu} \nabla p_{1}\right)=b_{11} \frac{\partial p_{1}}{\partial t}+\alpha_{1} \frac{\partial e}{\partial t}, \\
& G \nabla^{2} u_{i}+(G+\lambda) \frac{\partial e}{\partial x_{i}}=\alpha_{1} \frac{\partial p_{1}}{\partial x_{i}}
\end{aligned}
$$

where

$$
\begin{aligned}
b_{11} & =\phi_{1} c_{1}+\alpha_{1}\left(\beta_{1}-\alpha_{1}\right) c_{b}^{*}, \\
& =\phi_{1} c_{1}+\left(\alpha_{1}-\phi_{1}\right) c_{s} .
\end{aligned}
$$

Eqs. 28 through 30 agree with the single-porosity results presented by Chen et al. [1995].

If we let the matrix pore volume shrink to zero then $\phi_{1}=0, k_{1}=0$, and $c_{b}{ }^{*}=c_{s}$. It follows that $\alpha_{1}=\beta_{1}=0$ (see Eqs. A-9a and B-11a), $b_{11}=b_{12}=b_{13}=b_{21}=0, b_{23}=\alpha_{2}, b_{22}$ is still defined by Eq. 25e, and $\Gamma=0$. The result is the system given by Eqs. 28 through 30 but with the subscripts "1" and " 11 " replaced by " 2 " and " 22 ", respectively.

Finally, we may deduce single-porosity equations based on the condition that only one fluid-pressure field exists for a single-phase single-porosity system. Thus, letting $p_{1}=p_{2}=p$ in Eqs. 24 and 26 and simplifying give 


$$
\nabla \cdot\left(\frac{k_{t}}{\mu} \nabla p\right)=\left[\phi_{t} c+\left(\alpha_{t}-\phi_{t}\right) c_{s}\right] \frac{\partial p}{\partial t}+\alpha_{t} \frac{\partial e}{\partial t},
$$

and

$$
G \nabla^{2} u_{i}+(G+\lambda) \frac{\partial e}{\partial x_{i}}=\alpha_{t} \frac{\partial p}{\partial x_{i}}
$$

where $k_{t}=k_{1}+k_{2}, \phi_{t}=\phi_{1}+\phi_{2}, c=c_{1}=c_{2}$, and $\alpha_{t}=\alpha_{1}+\alpha_{2}$. In Eq. 31 , the coefficient of $\partial p / \partial t$ results from $\left(b_{11}+b_{12}+b_{21}+b_{22}\right)$ while the coefficient of $\partial e / \partial t$ results from $\left(b_{13}+b_{23}\right)$. The system given by Eqs. 31 and 32 basically is the same as the system of Eqs. 28 through 30, except that the former is in terms of total properties.

Single-Permeability/Dual-Porosity System. This is the system most often used in practice. For $k_{2}>>k_{1}$, we may assume $k_{1}=0$. Thus, the left-hand-sides of Eqs. 22a and 24a vanish. However, two fluid-pressure equations are still required to couple with deformation equation.

Incompressible Solid. The condition of incompressible solid is $c_{s}=0$. It follows that $\alpha_{1}=\beta_{1}=1$ and $\alpha_{2}=\beta_{2}=0$ (see Eqs. A-7, A-8, B-8, and B-10). The coefficients in Eq. 25 reduce to $b_{11}=\phi_{1} c_{1}, b_{12}=0, b_{13}=c_{b}{ }^{*} / c_{b}, b_{21}=0, b_{22}=\phi_{2} c_{2}$, while $b_{23}$ remains the same. Eqs. 24 and 26 then become

$$
\begin{aligned}
& \nabla \cdot\left(\frac{k_{1}}{\mu} \nabla p_{1}\right)=\phi_{1} c_{1} \frac{\partial p_{1}}{\partial t}+\frac{c_{b}^{*}}{c_{b}} \frac{\partial e}{\partial t}-\Gamma, \ldots \ldots \ldots . . . . . . \\
& \nabla \cdot\left(\frac{k_{2}}{\mu} \nabla p_{2}\right)=\phi_{2} c_{2} \frac{\partial p_{2}}{\partial t}+\left(1-\frac{c_{b}^{*}}{c_{b}}\right) \frac{\partial e}{\partial t}+\Gamma,
\end{aligned}
$$

and

$$
G \nabla^{2} u_{i}+(G+\lambda) \frac{\partial e}{\partial x_{i}}=\frac{\partial p_{1}}{\partial x_{i}}
$$

respectively.

It is interesting to note that only $p_{1}$ (the fluid-pressure in matrix blocks) affects the displacement equation (see Eq. 34). This observation seems to suggest that the rock deformation is controlled more by the fluid pressure in the matrix blocks than the fluid pressure in the fractures.

\section{CONCLUSIONS}

The major contribution of this paper is demonstrating how Biot's isothermal, linear poroelastic, coupled twophase (fluid and rock), single-porosity model can be extended to a dual-porosity system. The commonly used systematic fluid-flow modeling is preserved in our model development. This allows a better understanding of the relationship between the existing fluid-flow-only models and a coupled fluid-flow/geomechanics model.

The change of pore volumes is identified to be the most critical consideration in the coupling of fluid flow and rock deformation of a dual-porosity-based model. Interpretation of pore volumes in the context of the dual-porosity concept is proposed and incorporated into the coupled field equations. Continuity between single-porosity and dualporosity concepts is established by showing that the coupled fluid-flow/geomechanics single-porosity model is a limiting case of the proposed coupled dual-porosity model.

\section{NOMENCLATURE}

$$
\begin{aligned}
c & =\text { compressibility, } \mathrm{Lt}^{2} / \mathrm{m} \\
e & =\text { volume strain, dimensionless } \\
E & =\text { Young's modulus, } \mathrm{m} / \mathrm{Lt}^{2} \\
G & =\text { shear modulus, } \mathrm{m} / \mathrm{Lt}^{2} \\
k & =\text { permeability, } \mathrm{L}^{2}, \mathrm{md} \\
K & =\text { modulus, } \mathrm{m} / \mathrm{Lt}^{2} \\
p & =\text { pressure } \mathrm{m} / \mathrm{Lt}^{2} \\
t & =\text { time, } \mathrm{t} \\
u & =\text { displacement, } \mathrm{m} / \mathrm{Lt}^{2} \\
v & =\text { velocity, } \mathrm{L} / \mathrm{t} \\
V & =\text { volume, } \mathrm{L}^{3}
\end{aligned}
$$$$
x, y, z=\text { distance, } \mathrm{L}
$$

$\alpha=$ effective stress constant associated with the change of bulk volume, dimensionless

$\beta=$ effective stress constant associated with the change of pore volume, dimensionless

$\delta_{i j}=$ Kronecker's delta $\left(\delta_{i j}=1\right.$ for $i=j, \delta_{i j}=0$ for $\left.i \neq j\right)$

$\varepsilon=$ strain, dimensionless

$\phi=$ porosity, fraction

$\Gamma=$ interporosity flow, $\mathrm{L}^{3 / \mathrm{t} / \mathrm{L}^{3}}$

$\lambda=$ Lame's constant, $\mathrm{m} / \mathrm{Lt}^{2}$

$=3 v K_{b} /(1+v)=2 v G /(1-2 v)=K_{b}-(2 / 3) G$

$\mu=$ fluid viscosity, $\mathrm{m} / \mathrm{Lt}$

$v=$ Poisson's ratio, dimensionless

$\rho=$ density, $\mathrm{m} / \mathrm{L}^{3}$

$\sigma=$ stress, $\mathrm{m} / \mathrm{Lt}^{2}$

\section{Subscripts}

$$
\begin{aligned}
b & =\text { bulk } \\
m & =\text { mean } \\
n & =\text { index of primary and secondary pores } \\
p & =\text { pore } \\
s & =\text { solid } \\
t & =\text { total, system } \\
0 & =\text { reference } \\
1 & =\text { primary pores (matrix blocks) } \\
2 & =\text { secondary pores (fractures) }
\end{aligned}
$$




\section{Superscripts}

$e=$ effective

* = single-porosity system

\section{ACKNOWLEDGMENTS}

The authors thank Phillips Petroleum, Union Pacific Resources, Vastar Resources, Conoco, and Amoco for their support of this study.

\section{REFERENCES}

Bai, M., Elsworth, D., and Roegiers, J.-C.: "Multiporosity/Multi-permeability Approach to the Simulation of Naturally-Fractured Reservoirs," Water Resources Res. (1993) 29, 1621-1633.

Barenblatt, G.I., Zehltov, Iu.P., and Kochina, I.N.: "Basic Concepts in the Theory of Seepage of Homogeneous Liquids in Fissured Rocks (Strata)," Priklad. Mat. Mekh. (1960) 24, 852-864 (English translation in Soviet Appl. Math. Mech.).

Berryman, J.G.: "Effective Stress for Transport Properties of Inhomogeneous Porous Rock," J. Geophys. Res. (1992) 97, 17409-17424.

Beskos, D.E. and Aifantis, E.C.: "On the Theory of Consolidation With Double Porosity - II," Int. J. Engng. Sci. (1986) 24, 1697-1716.

Biot, M.A.: "General Theory of Three-Dimensional Consolidation," J. Appl. Phys. (1941) 12, 155-164.

Biot, M.A.: "Theory of Elasticity and Consolidation for a Porous Anisotropic Solid," J. Appl. Phys. (1955) 26, 182-185.

Biot, M.A.: "General Solutions of the Equations of Elasticity and Consolidation for a Porous Material," $J$. Appl. Mech. (1956) 27, 91-96.

Biot, M.A. and Willis, D.G.: "The Elastic Coefficients of the Theory of Consolidation," J. Appl. Mech. (1957) 24, 594-601.

Chen, H.-Y., Poston, S.W., and Raghavan, R.: "The Well Response in a Naturally Fractured Reservoir: Arbitrary Fracture Connectivity and Unsteady Fluid Transfer," paper SPE 20566 presented at the 1990 SPE Annual Technical Conference and Exhibition, New Orleans, LA, Sept. 23-26.

Chen, H.-Y., Teufel, L.W., and Lee, R.L.: "Coupled Fluid Flow and Geomechanics in Reservoir Study - I. Theory and Governing Equations," paper SPE 30752 presented at the 1995 SPE Annual Technical Conference and Exhibition, Dallas, TX, Oct. 22-25.

Duguid, J.O. and Lee, P.C.Y.: "Flow in Fractured Porous Media," Water Resources Res. (1977) 13, 558-566.
Geertsma, J.: "The Effect of Fluid Pressure Decline on Volumetric Changes of Porous Rocks," Trans. AIME (1957) 210, 331-340.

Khalili-Naghadeh, N. and Valliappan, S.: "Flow Through Fissured Porous Media With Deformable Matrix: Implicit Formulation," Water Resources Res. (1991) 27, 1703-1709.

Nur, A. and Byerlee, J.D.: "An Exact Effective Stress Law for Elastic Deformation of Rock With Fluids," $J$. Geophys. Res. (1971) 76, 6414-6419.

Teufel, L.W., Rhett, D.W., Farrell, H.E., and Lorenz, J.C.: "Control of Fractured Reservoir Permeability by Spatial and Temporal Variations in Stress Magnitude and Orientation," paper SPE 26437 presented at the 1993 SPE Annual Technical Conference and Exhibition, Houston, TX, Oct. 3-6.

Valliappan, S. and Khalili-Naghadeh, N.: "Flow Through Fissured Porous Media With Deformable Matrix," Int. J. Num. Meth. Eng. (1990) 29, 1079-1094.

Verruijt, A.: "Elastic Storage in Aquifers," Flow Through Porous Media, R.J.M. De Wiest (ed.), Academic, San Diego, California (1969) 331-376.

Wilson, R.K. and Aifantis, E.C.: "On the Theory of Consolidation With Double Porosity," Int. J. Engng. Sci. (1982) 20, 1009-1035.

\section{APPENDIX A - CHANGE OF BULK VOLUME}

The appendix describes the change of bulk volume and the associated effective stress law of a dual-porosity system.

Single-Porosity. The change of bulk volume of a singleporosity system can be described in terms of effective stress as [Chen et al., 1995]

$$
\mathrm{d} e=\frac{\mathrm{d} V_{b}}{V_{b}}=c_{b}^{*} \mathrm{~d} \sigma_{m}^{e},
$$

where the effective mean stress is defined as

$$
\sigma_{m}^{e}=\sigma_{m}+\alpha^{*} p
$$

Here, $c_{b}{ }^{*}$ is the bulk compressibility of a nonfractured rock, and $\alpha^{*}$ is the effective stress coefficient for the process of change of bulk volume. To avoid confusion with the later notations of a dual-porosity system, the superscript $*$ is used, if necessary, to refer a single-porosity system. $\alpha^{*}$ can be expressed as [Geertsma, 1957; Nur and Byerlee, 1971]

$$
\alpha^{*}=1-\frac{c_{s}}{c_{b}^{*}},
$$


where $c_{s}$ and $c_{b}{ }^{*}$ are the unjacketed bulk compressibility and drained jacketed compressibility, respectively. $\alpha^{*}$ is considered to be bounded by $\phi \leq \alpha^{*} \leq 1$ [Biot and Willis, 1957; Berryman, 1992].

Dual-Porosity. For a dual-porosity system, our approach is to maintain the functional form described by Eq. A-1 but modify the effective stress law given by Eq. A-2.

Since two pressure fields are presented in a general dual-porosity system, Eq. A-2 is extended naturally to

$$
\sigma_{m}^{e}=\sigma_{m}+\alpha_{1} p_{1}+\alpha_{2} p_{2}
$$

where $\alpha_{1}$ and $\alpha_{2}$ are the effective stress coefficients associated with $p_{1}$ and $p_{2}$, respectively. In other words, the change of bulk fractured rock volume is

$$
\mathrm{d} e=\frac{\mathrm{d} V_{b}}{V_{b}}=c_{b}\left(\mathrm{~d} \sigma_{m}+\alpha_{1} \mathrm{~d} p_{1}+\alpha_{2} \mathrm{~d} p_{2}\right)
$$

where $c_{b}$ is the bulk compressibility of a fractured rock.

Note that Eq. A-5 basically is the differential form of

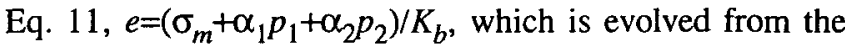
constitutive strain-stress relation (Eq. 9a). Eq. A-5 means that the contribution of fluid pressures $\left(p_{1}\right.$ and $\left.p_{2}\right)$ on the change of bulk volume are distributed or weighted according to effective stress coefficients, $\alpha_{1}$ and $\alpha_{2}$. Additionally, we require the following constraint on the sum of $\alpha_{1}$ and $\alpha_{2}$,

$$
\alpha_{t}=\alpha_{1}+\alpha_{2}=1-\frac{c_{s}}{c_{b}}
$$

such that Eq. A-2 (single-porosity) can be recovered from Eq. A-4 (dual-porosity) if the fractures are "dead" (e.g., healed fractures) or for the case of fully pressure equilibrium (i.e., $p_{1}=p_{2}=p$ ). Note that $c_{s}$ is measured under an unjacketed condition where the fluid is allowed to penetrate the connected pores. This condition suggests that $c_{s}$ should be independent of the presence of fractures. In other words, $c_{s}$ is the same for both single-porosity (nonfractured) and dual-porosity (tractured) systems.

For fractured rocks, it is reasonable to assume that the majority volume of the bulk volume is comprised of matrix blocks. Thus, it appears logically to approximate $\alpha_{1}$ (which is associated with the matrix blocks) as the $\alpha^{*}$ (Eq. A-3), i.e.,

$$
\alpha_{1} \cong \alpha^{*}=1-\frac{c_{s}}{c_{b}^{*}}
$$

$\alpha_{2}$ then can be deduced from Eqs. A-6 and A-7 which gives

$$
\alpha_{2}=\frac{c_{s}}{c_{b}^{*}}\left(1-\frac{c_{b}^{*}}{c_{b}}\right)=\left(1-\alpha_{1}\right)\left(1-\frac{c_{b}^{*}}{c_{b}}\right) .
$$

The proposed $\alpha_{1}$ and $\alpha_{2}$ (Eqs. A-7 and A-8) turn out to be the same as those presented by Wilson and Aifantis [1982]. Rationale and derivations, however, are not given by these authors.

Limiting Values of $\alpha_{\boldsymbol{n}}$. If we let the primary pore volume shrink to zero, i.e., $\phi_{1}=0$, then $c_{s}$ must be equal to $c_{b}{ }^{*}$, i.e., $c_{s}=c_{b}{ }^{*}$. Similarly, if $\phi_{2}=0$ then $c_{b}{ }^{*}=c_{b}$. With the above arguments, the limiting values of $\alpha_{1}$ (Eq. A-7) and $\alpha_{2}$ (Eq. A-8) corresponding to $\phi_{1}=0$ and $\phi_{2}=0$ are

$$
\phi_{1}=0\left(c_{s}=c_{b}^{*}\right): \quad \alpha_{1}=0 ; \quad \alpha_{2}=1-\frac{c_{s}}{c_{b}}=\alpha_{t},
$$

and

$$
\phi_{2}=0\left(c_{b}^{*}=c_{b}\right): \quad \alpha_{1}=1-\frac{c_{s}}{c_{b}}=\alpha_{t} ; \quad \alpha_{2}=0
$$

respectively.

Eq. A-7 suggests that $\alpha_{1}=1$ if $c_{s}=0$. Eq. A-8 suggests that $\alpha_{2}=0$ as long as $\alpha_{1}=1$ (e.g., $c_{s}=0$ ). It is expected that $c_{b}{ }^{*} \leq c_{b}$, and therefore $0 \leq\left[1-\left(c_{b} * / c_{b}\right)\right] \leq 1$. Eq. A-8 then suggests that $\alpha_{2}$ is bounded by $0 \leq \alpha_{2} \leq\left(1-\alpha_{1}\right) . \alpha_{2}=1-\alpha_{1}$ if $c_{b}^{*}<<c_{b}$.

\section{APPENDIX B - CHANGE OF PORE VOLUME}

The same approach used for bulk volume change is also applied to pore volume change in this section. The task, however, is more difficult for two reasons. First, two types of pore volumes (fractures and matrix blocks) are involved. Second, the functional form of pore volume change is more complicated. The notations adopted here follow those of Appendix A.

Single-Porosity. The following effective stress expression can be written for $\mathrm{d} V_{p} / V_{p}$ of a single-porosity system [Chen et al., 1995]

$$
\frac{\mathrm{d} V_{p}}{V_{p}}=\frac{\alpha^{*} c_{b}^{*}}{\phi} \mathrm{d} \sigma_{m}^{e}=\frac{c_{b}^{*}-c_{s}}{\phi} \mathrm{d} \sigma_{m}^{e},
$$

where the effective stress for this particular process is

$$
\sigma_{m}^{e}=\sigma_{m}+\beta * p
$$


and

$$
\beta^{*}=1-\frac{\phi c_{s}}{\alpha^{*} c_{b}^{*}}=1-\frac{\phi c_{s}}{c_{b}^{*}-c_{s}}
$$

$\beta^{*}$ is considered to be bounded by $\alpha \leq \beta^{*} \leq 1$ [Berryman, 1992].

Dual-Porosity. For a dual-porosity system, the effective stress is assumed to be

$$
\sigma_{m}^{e}=\sigma_{m}+\beta_{1} p_{1}+\beta_{2} p_{2}
$$

where two pressure fields $\left(p_{1}\right.$ and $\left.p_{2}\right)$ and two effective stress coefficients $\left(\beta_{1}\right.$ and $\beta_{2}$ ) are considered. We will assume that Eq. B-4 is applicable for all pore volume deformation processes including primary pore (matrix blocks), secondary pore (fracture), and total pore volumes.

For the change of total pore volume, the following relations are postulated which closely follows Eq. B-1,

$$
\frac{\mathrm{d} V_{p t}}{V_{p t}}=\alpha_{t} \frac{c_{b}}{\phi_{t}}\left(\mathrm{~d} \sigma_{m}+\beta_{1} \mathrm{~d} p_{1}+\beta_{2} \mathrm{~d} p_{2}\right),
$$

and the associated constraint,

$$
\beta_{t}=\beta_{1}+\beta_{2}=1-\frac{\phi_{t} c_{s}}{\alpha_{t} c_{b}}
$$

where $\alpha_{t}$ is defined by Eq. A- 6 . Eq. B-5 reduces to that of a single-porosity system in terms of total properties if $p_{1}=p_{2}=p$.

Extending the analysis of pore volume change of a single-porosity system in Chen et al., [1995] to a dualporosity system, the following form is assumed for the change of primary pore volume,

$$
\frac{\mathrm{d} V_{p 1}}{V_{p 1}}=\alpha_{1} \frac{c_{b}^{*}}{\phi_{1}}\left(\mathrm{~d} \sigma_{m}+\beta_{1} \mathrm{~d} p_{1}+\beta_{2} \mathrm{~d} p_{2}\right)
$$

where $\beta_{1}$ is

$$
\beta_{1}=1-\frac{\phi_{1} c_{s}}{\alpha_{1} c_{b}^{*}}=1-\frac{\phi_{1} c_{s}}{c_{b}^{*}-c_{s}},
$$

and $\alpha_{1}$ is defined by Eq. A-7.

Since $\mathrm{d} V_{p 2}=\mathrm{d} V_{p t}-\mathrm{d} V_{p 1}, \mathrm{~d} V_{p 2}$ can be derived from Eqs. $\mathrm{B}-5$ and B-7. The result is

$$
\frac{\mathrm{d} V_{p 2}}{V_{p 2}}=\left(1-\frac{c_{b}^{*}}{c_{b}}\right) \frac{c_{b}}{\phi_{2}}\left(\mathrm{~d} \sigma_{m}+\beta_{1} \mathrm{~d} p_{1}+\beta_{2} \mathrm{~d} p_{2}\right) .
$$

$\beta_{2}$ can be determined from Eqs. B-6 and B-8 which gives

$$
\beta_{2}=\frac{\phi_{1} c_{s}}{\alpha_{1} c_{b}^{*}}\left(1-\frac{\phi_{t} \alpha_{1} c_{b}^{*}}{\phi_{1} \alpha_{t} c_{b}}\right)=\left(1-\beta_{1}\right)\left(1-\frac{\phi_{t} \alpha_{1} c_{b}^{*}}{\phi_{1} \alpha_{t} c_{b}}\right) .
$$

Limiting Values of $\beta_{n}$. Similar to the approach used to obtain Eq. A-9, the limiting values of $\beta_{n}$ (Eqs. B-8 and B10) corresponding to $\phi_{1}=0$ and $\phi_{2}=0$ are

$$
\phi_{1}=0\left(c_{s}=c_{b}^{*}\right): \quad \beta_{1}=0 ; \quad \beta_{2}=1-\frac{\phi_{t} c_{s}}{\alpha_{t} c_{b}}=\beta_{t},
$$

and

$$
\phi_{2}=0\left(c_{b}^{*}=c_{b}\right): \quad \beta_{1}=1-\frac{\phi_{t} c_{s}}{\alpha_{t} c_{b}}=\beta_{t} ; \quad \beta_{2}=0,
$$

respectively.

Eq. B-8 suggests that $\beta_{1}=1$ if $c_{s}=0$. Eq. B-10 suggests the followings: (i) $\beta_{2}=0$ as long as $\beta_{1}=1$ (e.g., $c_{s}=0$ ), and (ii) $\beta_{2}=1-\beta_{1}$ if $\phi_{t} \alpha_{1} c_{b}{ }^{*}<<\phi_{1} \alpha_{t} c_{b}$.

Evaluating the changes of pore volumes (Eqs. B-7 and B-9) according to Eqs. A-9 and B-11 gives

$$
\frac{\mathrm{d} V_{p n}}{V_{p n}}=\alpha_{n} \frac{c_{b}}{\phi_{n}}\left(\mathrm{~d} \sigma_{m}+\beta_{n} \mathrm{~d} p_{n}\right),
$$

where $n=1$ if $\phi_{2}=0$ and $n=2$ if $\phi_{1}=0$. Eq. B- 12 basically is the same as the single-porosity/single-pressure equation given by Eq. B-1. 